\title{
Introduction and Farewell
}

\author{
Deborah Rose and Thom van Dooren
}

This issue of the Ecological Humanities is focussed on two of the greatest global phenomena that challenge contemporary social and cultural practice: the Anthropocene and climate change. In the first paper, Ben Dibley offers seven theses on the Anthropocene and 'attachment'. He takes up questions of time, history, politics, and the human. Nick Mansfield presents an extended review essay that engages with the challenges of climate change. His essay starts with the prophetic words: 'climate change will also bring with it problems to do with political time and historical time...'. In their contribution to this issue, Alice Robinson and Dan Tout bring their experience of drought into reflections on floods and belonging in the unique climate and culture of settler Australia. They explore what it means to call a place a 'home', and the need for settler societies to unsettle their own histories, ideas and relationships to produce more honest and sustainable possibilities for the future. These articles are all situated within an awareness of the far-more-than-human significance of events in this era of rapid change.

Meteorologists are telling us that La Niña will soon loosen her grip on Australia, and as El Niño gathers strength, we are likely to be entering another period of drought and-inevitably-fire. In light of climate change, unsettled expectations, challenging time concepts, and the near certainty of future bushfires in the 'sunburnt country', we asked Stephen Pyne to write an essay about fire in both its loved and unloved personae. Along with this reflective essay, we also offer a review of a new book by Matt Hall, Plants as Persons (SUNY Press).

We are experiencing both sweet and sad emotions as we announce that this will be the final issue of the Australian Humanities Review to contain a dedicated Ecological Humanities section. Over the past few years we have been closely monitoring the growth of the field of research that thrives under the rubrics of ecological and environmental humanities, and have come to the conclusion that there is a need for a new, international, open-access journal to publish outstanding scholarship that draws humanities disciplines into conversation with each other and with the natural and social sciences. With this context in mind, it is our delight to announce the establishment of a new journal: Environmental Humanities. The first issue of this new journal will appear in November 2012, with two issues following in each subsequent calendar year, continuing the pattern established in this journal < http://www.environmentalhumanities. org $>$. 
Libby Robin and Deborah Bird Rose launched the Ecological Humanities section of the Australian Humanities Review in 2004 with the aim of ensuring that research addressing a blind spot in contemporary thought would have a wellranked publication site. This blind spot was the place where ecological sciences and the humanities intersect. We launched the new journal section with an invitation (Rose and Robin 2004) to engage with the Ecological Humanities. In 2009 Thom van Dooren replaced Robin as co-editor.

The urgency of developing research excellence in the area of this intersection arises from the Anthropocene, and since our first issue in 2004 the need for this research has only grown. Over the course of a decade of publishing, we have offered several special issues, along with numerous original articles and reprints of a few 'classic essays'. The special issues include:

- 2011 'Unloved Others: Death of the disregarded in the time of extinctions', AHR 50.

- 2006 'Ecopoetics and the Ecological Humanities in Australia', AHR 39-40.

- 2005 'Desert Gardens: Waterless lands and the problems of adaptation', AHR 36.

- 2005 'Gregory Bateson and Ecological Aesthetics', AHR 35.

We would like to take this opportunity to thank all of the authors who have contributed to the Ecological Humanities, and all of the readers who have found intellectual sustenance in these pages. It is a pleasure to offer a warm invitation to continue these conversations in the coming years in a new international forum: Environmental Humanities. 